\title{
METHADIS: Methodology for the Design of Adaptive Hypermedia Systems for Learning based on Learning and Cognitive Styles ${ }^{*}$
}

\author{
Marcela Prieto \\ Universidad de Antofagasta \\ Departamento de Educación \\ Chile \\ mprieto@uantof.cl
}

\begin{abstract}
The design of eLearning systems aimed at personalizing the learning process, and which are based on given learning and cognitive styles, implies, from the teaching perspective, a need for evaluating potential teaching environments adapted to the preferences and forms of information processing of the students. In order to systematize this task, we have developed a methodology METHADIS which, based on a leaning objective, and by means of following a sequence of steps, determines instructional strategies custom-design for the specific needs of different users. Thus the contents, information formats, and activities are presented as a function of the learning styles; the helps and navigational options are given as a function of the specifics of each cognitive style. METHADIS was applied to a case of study where performance was compared between students in an experimental group which interacted with a hypermedia system design using this methodology and that of a control group.
\end{abstract}

\section{Introduction}

There are a great variety models and theories in the literature regarding learning styles and cognitive styles. Although some authors do not distinguish between learning styles and cognitive styles, there are others who clearly do [1].

In spite of their differences, the two styles have been used exclusively as a basis for adaptation of some Adaptive Hypermedia Systems (AHS). Adaptive Hypermedia Educational Systems (AHES) have been developed solely on the learning styles of their potential users, based on different theories, and adapting their presentation to the contents, and in some cases, to navigational options, too. Other systems,

\author{
Francisco García \\ Universidad de Salamanca \\ Departamento de Informática y Automática \\ España \\ fgarcia@usal.es
}

which have been based solely on adaptation to cognitive styles, have adapted navigational options.

METHADIS is a methodological proposal for the design, from the teaching perspective, of AHES based concurrently on learning styles and cognitive styles.

\section{METHADIS}

Developing a methodology for designing AHES based on learning and cognitive styles, it is necessary to incorporate elements related to the instructional design, in order to have a coherent system, with the objective of the learning - [2], and the characteristics of the styles, utilized. METHADIS envisions the process in three levels (Figure 1), which include a sequence of different activities which are interrelated.

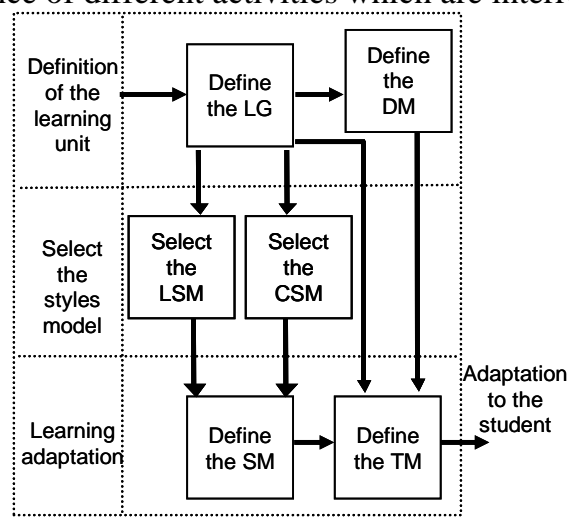

Figure 1. Methadis

The first level represents the definition of the learning unit of the system. This is formed of two activities. The first activity is the definition of the type of learning, which is the establishment of the purpose to be attained, by establishing a learning goal (LG). The teacher who designs the learning objective must clearly specify its purpose. For this, the following tasks must be carried out: selection of the domain of

\footnotetext{
* We thank the University of Antofagasta for its constant support. This work has been partly financed by the Spanish Ministry of Education and Science as well as FEDER KEOPS Project (TSI2005-00960).
} 
learning, choice of the taxonomy that will be used, and defining the specific type of learning. Beginning from the learning objective in the Domain Model (DM), the conceptual structure of the domain of the learning is portrayed, its contents are detailed, and the structure is organized.

On the second level, selection is made from theories of learning styles and of cognitive styles. For this, two activities are defined, including that of the selection of these theories of styles. Once the selection has been carried out, measuring instruments must be applied to the students who will be the users of the system.

On the last level, which is that of adaptation of the teaching, the categories of the users must be identified, with their respective forms of adaptation. The Student Model (SM), in this specific case, will be a model based on stereotypes. To define all the possible categories of users, one must obtain the Cartesian product between the group composed of all the categories of learning styles, together with the group of cognitive styles.

To establish the instructional strategies that will be used, the Teaching Model (TM) is defined. All the preceding activities converge in this model [3]. These teaching strategies are established through two forms of adaptation, including: the presentation of content as a function of learning styles, and navigational options adjusted to cognitive styles.

\section{Case study}

METHADIS was applied to design a prototype for a hypermedia system adapted to learning and cognitive styles of a group of university students in the teaching curricula in Elementary Education, and Biology \& Sciences, in the Faculty of Education, University of Antofagasta, Chile.

The design of the experiment included pre-and post-testing, and a control group. The experimental, or independent variable was the hypermedia system adapted to the learning and cognitive styles. A neutral treatment was applied to the control group. The sample was formed of 46 students who were randomly assigned to the experimental group (26) and to the control group (20).

The material established in the teaching model was fixed in the hypermedia system, provided through the Claroline content generator, such that each student in the experimental group interacted with the system content, which was created as a function of their styles. In contrast, all the students of the control group subscribed to the same hypermedia system, structured solely on the basis of conceptual relations with the contents, without considering other factors.

Five types of comparisons were obtained, within a confidence level of $95 \%$. In the first comparison, referent to the results obtained in the pre-tests of the experimental and control groups, it was determined that there were no statistical differences between the results of the pre-tests applied, from which it was deduced that the control and experimental groups were sufficiently homogeneous regarding pre-existing knowledge.

In order to compare mean values obtained between the post-test and pre-test of each group, it was determined that in both there were statistically significant differences, confirming that learning had indeed occurred. Comparisons of post- test results of both groups, however, showed statistically significant differences, in which the experimental group had achieved a greater average degree of learning. The same results were obtained by comparing the differences between the post-test and pre-test results between the experimental and control groups. From the above it was deduced that the treatment given to the experimental group was more effective, in that it achieved a better learning result, not attributable to chance.

\section{Conclusions}

METHADIS, as a proposal, requires a greater number of tests in the future, with larger numbers of participating students, to be able to improve and validate it through a feedback process.

It is also necessary to be able to compare the effect of adaptation on the presentation of contents based on learning styles, with the effect of adaptation to navigational options based on cognitive styles.

This is therefore not the expression of a limited methodology, but rather one which is capable of evolving into new directions of future study.

\section{References}

[1] Jonassen, D., Grabowski, B., Handbook of individual differences, Lawrence Erlbaum Associates, Hillsdale, NJ, 1993.

[2] Merrill, M. D., "Instructional strategies and learning styles: Which takes precedence?", In R. A. Reiser \& J. V. Dempsey (Eds.), Trends and Issues in Instructional Technology, Prentice Hall, Columbus, OH., 2001, 99-106.

[3] Prieto, M., Leighton, H., García, F., "Adaptive Educational Hypermedia Proposal base on learning styles", In P. de Bra, W. Nejdl (Eds.) Adaptive Hypermedia and Adaptive Web-Based Systems, LCCS 3137, AH 2004, Springer, Berlin, 2004, 316-319. 